\title{
Theoretical Analysis Reveals Cost and Benefit of Proofreading in Coronavirus Genome Replication
}

\author{
Joel D. Mallory, ${ }^{1}$ Xian F. Mallory, ${ }^{2}$ Anatoly B. Kolomeisky, ${ }^{1,3,4,5}$ and Oleg A. Igoshin ${ }^{1,3,6,7}$ \\ ${ }^{1}$ Center for Theoretical Biological Physics, Rice University, Houston, TX 77005 \\ ${ }^{2}$ Department of Computer Science, Florida State University, Tallahassee, FL 32306 \\ ${ }^{3}$ Department of Chemistry, Rice University, Houston, TX 77005 \\ ${ }^{4}$ Department of Chemical and Biomolecular Engineering, Rice University, Houston, TX 77005 \\ ${ }^{5}$ Department of Physics and Astronomy, Rice University, Houston, TX 77005 \\ ${ }^{6}$ Department of Bioengineering, Rice University, Houston, TX 77005 \\ ${ }^{7}$ Department of Biosciences, Rice University, Houston, TX 77005
}

\section{CONTENTS}

Definitions and Equations of the Characteristic Properties for the SARS-CoV

RNA-Dependent RNA Polymerase Complex $\quad$ S2

Forward Master Equation Formalism $\quad$ S2

Definitions of the Characteristic Properties $\quad$ S3

Kinetic Parameters $\quad$ S4

$\begin{array}{ll}\text { Sensitivity Analysis: Robustness of Conclusions } & \text { S7 }\end{array}$

Interplay of the Probabilities with the Pol-Exo Sliding Rate S11

Bioinformatics Calculations: Mutation Probability and Effective Speed S12

Interplay between Proofreading Cost and Effective Speed S16

$\begin{array}{ll}\text { Effective Speed Distribution } & \text { S18 }\end{array}$

Rankings the Characteristic Properties $\quad$ S19

$\begin{array}{ll}\text { References } & \text { S21 }\end{array}$ 


\section{DEFINITIONS AND EQUATIONS OF THE CHARACTERISTIC PROPERTIES FOR THE SARS-COV RNA-DEPENDENT RNA POLYMERASE COMPLEX}

\section{Forward Master Equation Formalism}

For an arbitrary biological system, there is a chemical kinetic network (kinetic mechanism) that consists of quasi-first-order-transitions between $N$ biochemical states with the rate constants $k_{i, j}$ corresponding to the elementary reactions $i \rightarrow j$. This is often referred to as a linear framework because the resulting kinetic equations are linear[1]. In general, there are multiple elementary reactions between the biochemical states $i$ and $j$ such that

$$
k_{i, j}=\sum_{\omega} k_{i, j}^{\omega}
$$

where $\omega$ denotes the index for the different reactions connecting the states $i$ and $j$. We can solve the set of forward master equations in the stationary state subject to the normalization condition:

$$
\mathbf{K} \cdot \mathbf{P}=\mathbf{0} \text { and } \mathbf{1}^{T} \cdot \mathbf{P}=1
$$

where 1 is an $N \times 1$ unit vector, and the is an $N \times N$ rate matrix:

$$
K_{j, i}= \begin{cases}k_{i, j}, & \text { for } j \neq i \\ -\sum_{i \neq j} k_{i, j}, & \text { for } j=i\end{cases}
$$

Specifically, for the SARS-CoV RNA-dependent RNA polymerase (RdRp) complex the rate matrix $\mathrm{K}$ is $5 \times 5$ and takes the following form:

$$
\mathbf{K}=\left(\begin{array}{ccccc}
-D_{1} & k_{-1, R}+k_{p, R} & k_{3, R} & k_{-1, W}+k_{p, W} & k_{3, W} \\
k_{1, R}+k_{-p, R} & -D_{2} & k_{-2, R} & 0 & 0 \\
k_{-3, R} & k_{2, R} & -D_{3} & 0 & 0 \\
k_{1, W}+k_{-p, W} & 0 & 0 & -D_{4} & k_{-2, W} \\
k_{-3, W} & 0 & 0 & k_{2, W} & -D_{5}
\end{array}\right)
$$




$$
\begin{aligned}
& D_{1}=\left(k_{1, R}+k_{1, W}+k_{-3, R}+k_{-3, W}+k_{-p, R}+k_{-p, W}\right) \\
& D_{2}=\left(k_{-1, R}+k_{2, R}+k_{p, R}\right) \\
& D_{3}=\left(k_{-2, R}+k_{3, R}\right) \\
& D_{4}=\left(k_{-1, W}+k_{2, W}+k_{p, W}\right) \\
& D_{5}=\left(k_{-2, W}+k_{3, W}\right) .
\end{aligned}
$$

Assuming this matrix is not rank deficient, Eq. (S2) has a unique solution for the vector of stationary probabilities $\mathrm{P}$ :

$$
\mathbf{P}=\left[P_{1}, P_{2} \ldots, P_{N}\right]^{T} .
$$

The stationary fluxes in the forward framework are defined as follows:

$$
J_{i, j}^{\omega}=k_{i, j}^{\omega} P_{i} .
$$

Here, $k_{i, j}$ is the rate constant and $P_{i}$ is the stationary probability of the state $i$ on the chemical kinetic network. The superscript $\omega$ denotes the possibility of multiple pathways connecting the states $i$ and $j$. For consistency of notation, we can drop the $\omega$ superscript and denote the stationary fluxes for the right $R$ and wrong $W$ nucleotides as follows with the rate constants denoted by $k_{i, R / W} \equiv k_{i, j}$ and $k_{-i, R / W} \equiv k_{j, i}$, and the stationary probabilities denoted by $P_{-i} \equiv P_{j}(\mathrm{i}=1,2,3, p)$ :

$$
J_{ \pm i, R / W}=k_{ \pm i, R / W} P_{ \pm i}
$$

In addition, we define all of the net fluxes (i.e., currents) in the following way:

$$
J_{i}=k_{i, R / W} P_{i}-k_{-i, R / W} P_{-i}
$$

\section{Definitions of the Characteristic Properties}

In the forward master equation formalism, the error rate $\eta$ is defined as the ratio of the product formation flux to create the wrong $W$ product to the product formation flux to create the right $R$ product:

$$
\eta=\frac{J_{W}}{J_{R}}
$$


The speed $V$ is defined as the right product formation flux such that $V=J_{R}$ instead of $V=J_{R}+J_{W}$ because $J_{R}>>J_{W}$. The proofreading cost $C$ is defined as the ratio of the proofreading flux to the product formation flux (i.e., the fraction of additional nucleotide triphosphate $[\mathrm{NTP}]$ molecules hydrolyzed during futile resetting cycles) in the steady-state:

$$
C=\frac{J_{\text {proof }}}{J_{\mathrm{P}}}=\frac{J_{3, R}+J_{3, W}}{J_{p, R}+J_{p, W}}
$$

Here, the total proofreading flux is given by,

$$
J_{\text {proof }}=J_{3, R}+J_{3, W}=k_{3, R} P_{\mathrm{ER} *}-k_{-3, R} P_{\mathrm{E}}+k_{3, W} P_{\mathrm{EW} *}-k_{-3, W} P_{\mathrm{E}},
$$

and the total product formation flux is given by,

$$
J_{\mathrm{P}}=J_{p, R}+J_{p, W}=k_{p, R} P_{\mathrm{ER}}-k_{-p, R} P_{\mathrm{E}}+k_{p, W} P_{\mathrm{EW}}-k_{-p, W} P_{\mathrm{E}} .
$$

\section{KINETIC PARAMETERS}

The kinetic proofreading (KPR) mechanism for the SARS-CoV RdRp complex is shown in Fig. S1 with all of the rate constants (kinetic parameters) for each elementary reaction. The kinetic parameters $k_{ \pm i, R}$ and the corresponding selectivities (discrimination factors) $f_{ \pm i}=k_{ \pm i, W} / k_{ \pm i, R}$ for each step are listed in Table. S1. All of the discrimination factors $f_{ \pm i}$ were held fixed during variation of the kinetic parameters during local and global parameter sampling. Moreover, the native error rate of the SARS-CoV RdRp complex was constrained to the experimental error rate $\eta_{\text {nat }}=3.4 \times 10^{-6}[2-4]$ in the KPR model. This was done by setting the rate constant $k_{p, W}$ for incorporation of a wrong $W$ nucleotide, and hence, the discrimination factor $f_{p}=k_{p, W} / k_{p, R}$. The kinetic parameter $k_{p, W}$ was chosen to constrain the error rate to the native one because the error rate is primarily controlled by the discrimination factor of the catalytic step $f_{p}$. Thus, the analytic expression for the error rate is proportional to the discrimination factor $f_{p}$ in Eq. (S12)):

$$
\eta=f_{p} \frac{\left(k_{-2, R}\left(k_{p, R}+k_{-1, R}\right)+k_{3, R}\left(k_{p, R}+k_{-1, R}+k_{2, R}\right)\right)\left(\left(k_{-2, W}+k_{3, W}\right)\left(k_{-p, W}+k_{1, W}\right)+k_{-3, W} k_{-2, W}\right)}{\left(\left(k_{-2, R}+k_{3, R}\right)\left(k_{-p, R^{+}} k_{1, R}\right)+k_{-3, R^{k}} k_{-2, R}\right)\left(k_{-2, W}\left(k_{p, W}+k_{-1, W}\right)+k_{3, W}\left(k_{p, W}+k_{-1, W}+k_{2, W}\right)\right)} .
$$




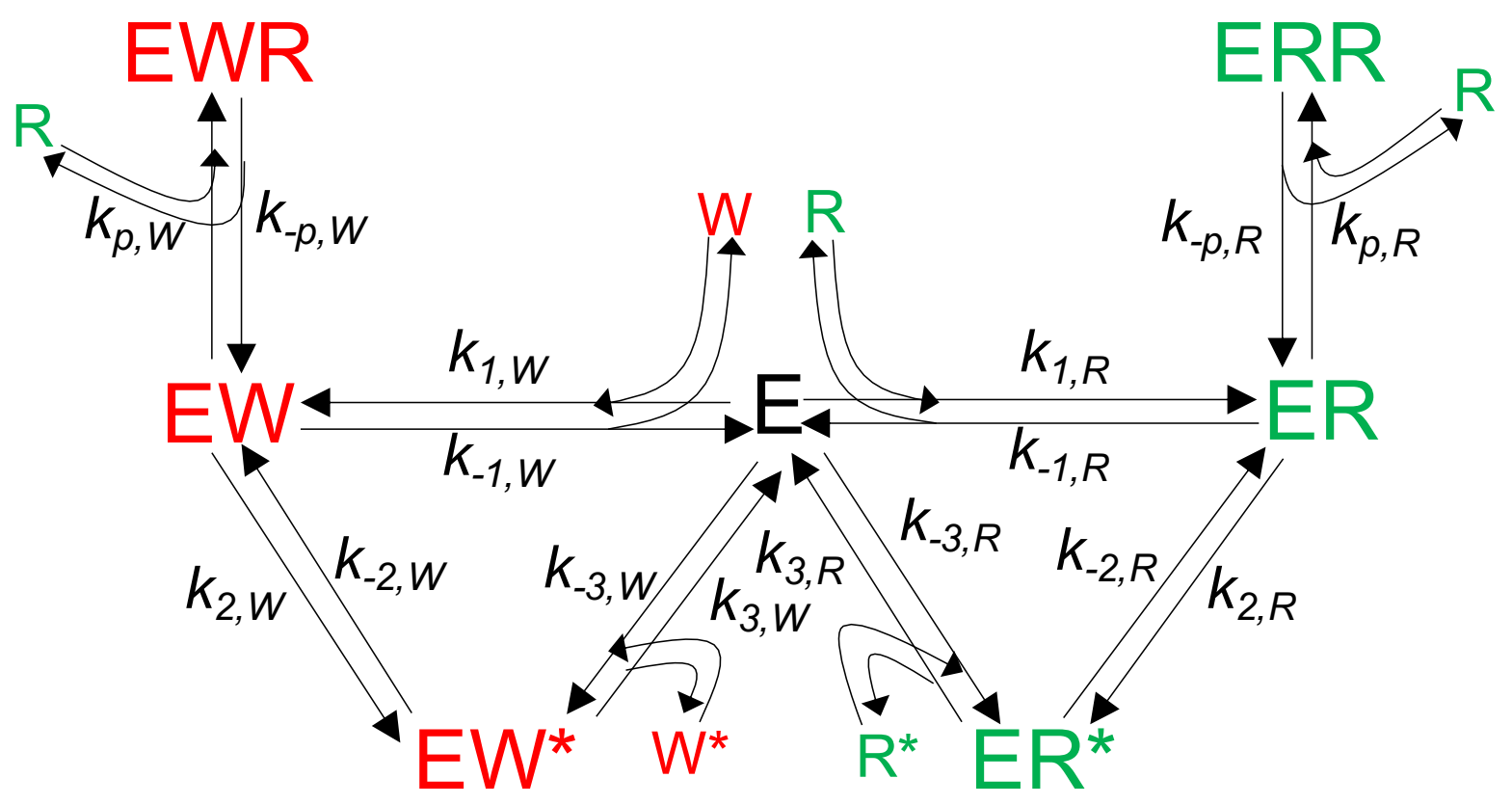

FIG. S1. Kinetic mechanism for the SARS-CoV RdRp complex with proofreading. All of the elementary reactions between the biochemical states are reversible. In the free enzyme state $E$, the RdRp complex can add either the right $R$ (green) or wrong $W$ (red) nucleotide to the growing chain. The RdRp complex can then proceed by adding another right $R$ nucleotide to the chain to form the products $E R R$ or $E W R$, or it can remove the nucleotide during proofreading via the sliding and hydrolysis steps, i.e., $E R / E W \rightarrow E R^{*} / E W^{*}$ and $E R^{*} / E W^{*} \rightarrow E$, respectively. 
TABLE S1. Experimental rate constants $k_{ \pm i, R}$ and corresponding discrimination factors $f_{ \pm i}$ for the SARS-CoV RdRp complex from Refs. 5-10. All of the rate constants are first order (or pseudofirst-order) in units of $\mathrm{s}^{-1}$. The rate constants $k_{-3, R}$ and $k_{-p, R}$ were determined from the chemical potential differences $\Delta \mu_{\mathrm{NTP}}=29.5 k_{B} T$ and $\Delta \mu_{\mathrm{p}}=9.0 k_{B} T$, respectively (see Eq. (S13)). The discrimination factors $f_{-3}$ and $f_{-p}$ were determined from a secondary constraint (see Eq. (S14))

\begin{tabular}{|c|c|c|}
\hline Kinetic Parameters & Value $\left(\mathrm{s}^{-1}\right)$ & Source \\
\hline$k_{1, R}$ & 120 & 5 \\
$k_{-1, R}$ & 1.3 & 6 \\
$k_{2, R}$ & 0.2 & $7-10$ \\
$k_{-2, R}$ & 700 & $7-10$ \\
$k_{3, R}$ & 900 & $7-10$ \\
$k_{-3, R}$ & $3.6 \times 10^{-12}$ & - \\
$k_{p, R}$ & 120 & 5 \\
$k_{-p, R}$ & 1.3 & 6 \\
$f_{1}$ & $8.3 \times 10^{-5}$ & 5,6 \\
$f_{-1}$ & $3.6 \times 10^{-5}$ & 6 \\
$f_{2}$ & 12 & $7-10$ \\
$f_{-2}$ & 1 & $7-10$ \\
$f_{3}$ & 1 & $7-10$ \\
$f_{-3}$ & 27 & - \\
$f_{p}$ & $4.0 \times 10^{-4}$ & 5 \\
$f_{-p}$ & $9.3 \times 10^{-4}$ & - \\
\hline
\end{tabular}

Due to the lack of kinetic experiments done on the SARS-CoV RdRp complex and the rapidly evolving nature of this field, we obtained the kinetic rates $k_{p m i, R / W}$ from different sources. One of the rate constants comes directly from kinetic data for the SARS-CoV RdRp complex, while the others are extrapolated kinetic data from other viral polymerases. The polymerization rate constant $k_{1, R}=k_{p, R}=120 \mathrm{~s}^{-1}$ is an average of the experimental rates for the SARS-CoV RdRp complex recently obtained for an annealed primer template $(90 \pm 4$ $\mathrm{s}^{-1}$ ) and for a hairpin loop template $\left(150 \pm 30 \mathrm{~s}^{-1}\right)[5]$. The reverse (pyrophosphorolysis) rate constant $k_{-1, R}=k_{-p, R}=1.3 \mathrm{~s}^{-1}$ was determined from kinetic experiments on the Poliovirus 
RdRp (3Dpol)[6]. The Pol-Exo sliding rate constants $\left(k_{2, R}=0.2 \mathrm{~s}^{-1}, k_{-2, R}=700 \mathrm{~s}^{-1}\right.$, and $k_{3, R}=900 \mathrm{~s}^{-1}$ ) were obtained from kinetic experiments[7] and theoretical papers on the T7 DNA polymerase[8-10].

During local and global variation of the kinetic parameters, the chemical potential difference $\Delta \mu_{\mathrm{NTP}}=29.5 k_{B} T$ was fixed at its physiological value for hydrolysis of one nucleotide triphosphate (NTP) molecule (NTP $\rightarrow \mathrm{NMP}+\mathrm{PP}_{i}$ ) by the SARS-CoV RdRp complex. The chemical potential difference for phosphodiester bond formation was also fixed at $\Delta \mu_{\text {phosphodiesterbond }}=9.0 k_{B} T$. The chemical potential differences over the cycles in the KPR mechanism are given by:

$$
\Delta \mu_{\mathrm{cycle}}=\ln \left(\prod_{i=1}^{N} \frac{k_{i, R}}{k_{-i, R}}\right)=\ln \left(\prod_{i=1}^{N} \frac{k_{i, W}}{k_{-i, W}}\right) .
$$

Since the cycles have the same chemical potential differences for right $R$ and wrong $W$ nucleotides, this leads to the following constraint on the discrimination factors:

$$
\prod_{i=1}^{N} \frac{f_{i}}{f_{-i}}=1 .
$$

\section{SENSITIVITY ANALYSIS: ROBUSTNESS OF CONCLUSIONS}

We performed the sensitivity analysis to test the robustness of our conclusions to variations of the kinetic parameters $k_{ \pm i, R}$ listed in Table. S1. To this end, we randomly sampled 200 sets of all the kinetic parameters from uniform distributions within the respective ranges of $\left[0.5 k_{ \pm i, R}, 2 k_{ \pm i, R}\right]$ and $\left[3 k_{ \pm i, R}, 6 k_{ \pm i, R}\right]$ while keeping all of the discrimination factors $f_{i}$ fixed. The first range was selected to look at the robustness of the characteristic properties (i.e., error rate $\eta$, speed $V$ [shown as the speed ratio $V / V_{\max }$, and proofreading $\operatorname{cost} C$ ) to smaller variations of the kinetic parameters. The second range was selected because it includes the range of physiological polymerization rate constants $600-700 \mathrm{~s}^{-1}$ proposed by Shannon et al.[5]. On a qualitative note, the characteristic properties from random sampling of the kinetic parameters (green curves) on the range $\left[0.5 k_{ \pm i, R}, 2 k_{ \pm i, R}\right]$ in Fig. S2A-C do not appear to deviate significantly from the curve calculated using the model kinetic parameters (black curve) from Table. S1. For the range $\left[3 k_{ \pm i, R}, 6 k_{ \pm i, R}\right]$ in Fig. S3A-C, the curves from random sampling are all shifted somewhat to the right (i.e., to faster Pol-Exo sliding rates $k_{2, R}$ ) as expected for the larger rate constants (lower kinetic barriers). However, even for 
this physiological range, the shape of the green curves does not change significantly from the black curve calculated using the model kinetic parameters from Table. S1. Thus, these qualitative results show that our conclusions for the SARS-CoV RdRp complex appear to be robust to variations of the kinetic parameters.

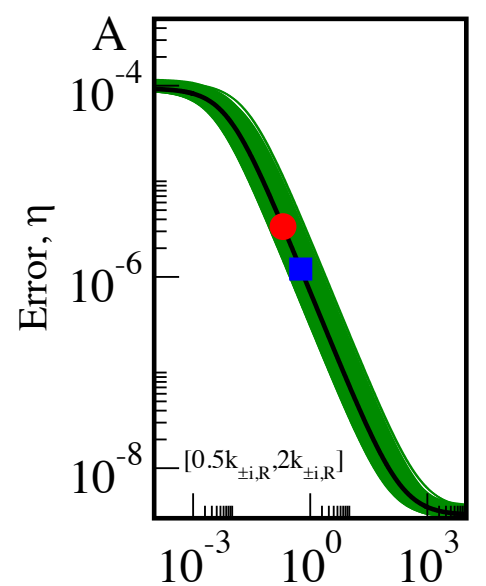

Pol-Exo Slding Rate, $k_{2, R}\left(\mathrm{~s}^{-1}\right)$

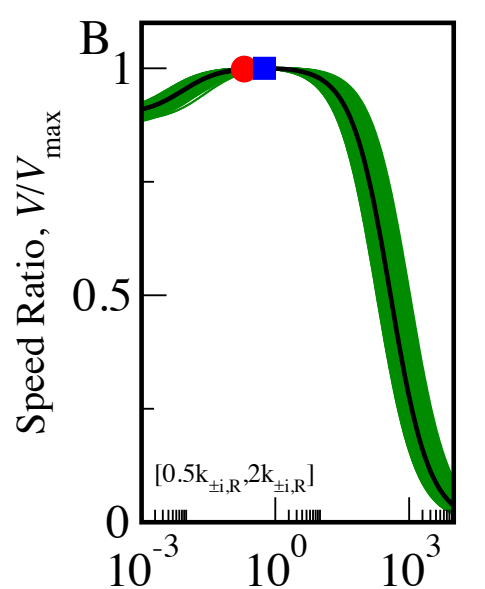

Pol-Exo Sliding Rate, $k_{2, R}\left(\mathrm{~s}^{-1}\right)$

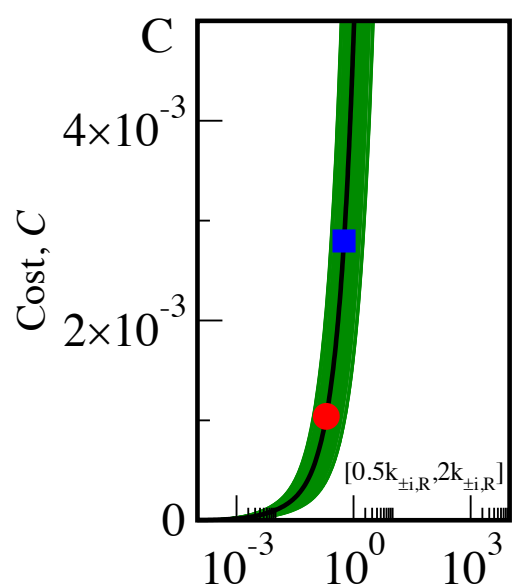

Pol-Exo Sliding Rate, $k_{2, R}\left(\mathrm{~s}^{-1}\right)$

FIG. S2. Curves from random sampling of 200 kinetic parameter sets from a uniform distribution between $\left[0.5 k_{ \pm i, R}, 2 k_{ \pm i, R}\right]$. All of the kinetic parameters from the KPR model were sampled from the uniform distribution. The black curves are the native model system with kinetic parameters from Table. S1. The green curves come from the random sampling. A. Error rate $\eta$ as a function of the Pol-Exo sliding rate $k_{2, R}$. B. Speed ratio $V / V_{\max }$ as a function of the Pol-Exo sliding rate $k_{2, R}$. C. Cost $C$ as a function of the Pol-Exo sliding rate $k_{2, R}$. 


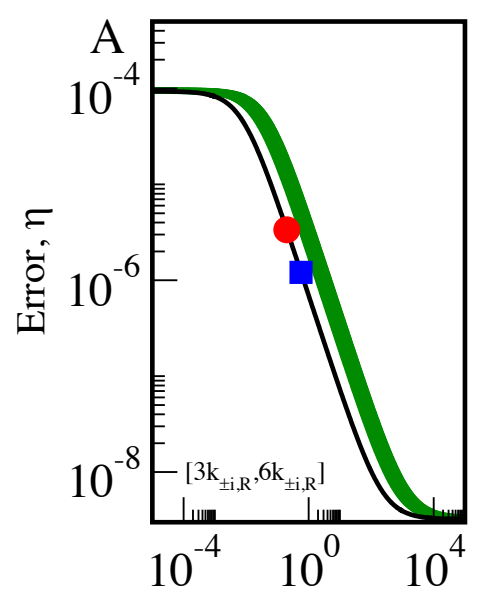

Pol-Exo Sliding Rate, $k_{2, R}\left(\mathrm{~s}^{-1}\right)$

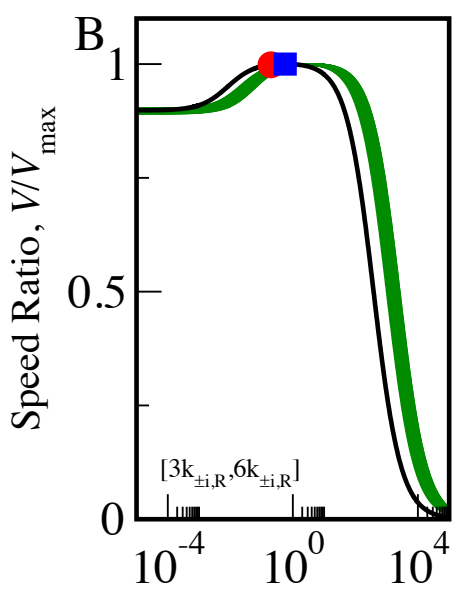

Pol-Exo Sliding Rate, $k_{2, R}\left(\mathrm{~s}^{-1}\right)$

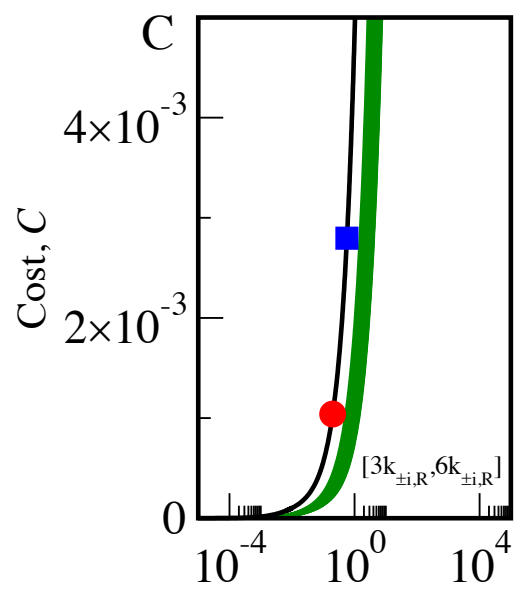

Pol-Exo Sliding Rate, $k_{2, R}\left(\mathrm{~s}^{-1}\right)$

FIG. S3. Curves from random sampling of 200 kinetic parameter sets from a uniform distribution between $\left[3 k_{ \pm i, R}, 6 k_{ \pm i, R}\right]$. All of the kinetic parameters from the KPR model were sampled from the uniform distribution. The black curves are the native model system with kinetic parameters from Table. S1. The green curves come from the random sampling. A. Error rate $\eta$ as a function of the Pol-Exo sliding rate $k_{2, R}$. B. Speed ratio $V / V_{\max }$ as a function of the Pol-Exo sliding rate $k_{2, R}$. C. Cost $C$ as a function of the Pol-Exo sliding rate $k_{2, R}$.

Furthermore, we performed the sensitivity analysis on the kinetic parameters in Table. S1 to test the robustness of our conclusions from a quantitative angle. Specifically, we examined the following conclusions: (1) the native error rate is far from the minimum error rate, but the native error rate is still much smaller than the error rate with no proofreading; (2) the speed is nearly optimized; (3) the proofreading cost at the maximum speed is much larger than the native cost. To check the robustness of the three conclusions, we evaluated the cumulative histograms of the characteristic properties for the kinetic parameter sets generated by random sampling in the ranges of $\left[0.5 k_{ \pm i, R}, 2 k_{ \pm i, R}\right]$ and $\left[3 k_{ \pm i, R}, 6 k_{ \pm i, R}\right]$. In addition, we computed the fraction of kinetic parameter sets that have the error rate, speed, and cost that satisfy the following conditions: (1) $\left(\eta_{\text {nat }}-\eta_{\min }\right) /\left(\eta_{0}-\eta_{\min }\right)<\epsilon_{\eta} ;(2)\left(V_{\max }-\right.$ $\left.V_{\text {nat }}\right) / V_{\max }<\epsilon_{V} ;(3)\left(C_{V_{\max }}-C_{\text {nat }}\right) / C_{V_{\max }}>\epsilon_{C}$. Here, the terms $\epsilon_{\eta}, \epsilon_{V}$, and $\epsilon_{C}$ are the chosen threshold conditions (i.e., tolerances) for computing the fraction of kinetic parameter sets that satisfy each of the three inequalities. The cumulative histograms for the characteristic properties of the kinetic parameter sets sampled in the $\left[0.5 k_{ \pm i, R}, 2 k_{ \pm i, R}\right]$ and $\left[3 k_{ \pm i, R}, 6 k_{ \pm i, R}\right]$ ranges are shown in Fig. S4A-C and Fig. S5A-C, respectively. 
The quantitative analysis predicts that our conclusions on the error rate and speed are robust despite the variations in the kinetic parameters in both ranges. In fact, $\sim 96 \%$ of the kinetic parameter sets satisfy the respective inequalities for the error rate and speed with $\epsilon_{\eta} \sim 0.1$ or lower and $\epsilon_{V} \sim 0.01$ or lower. Interestingly, the least robust of the conclusions is the one for the proofreading cost, where $\sim 96 \%$ of the kinetic parameter sets satisfy the inequality with $\epsilon_{C} \sim 0.4$ or lower. Overall, our quantitative results for the physiological parameter range $\left[3 k_{ \pm i, R}, 6 k_{ \pm i, R}\right]$ appear to be more robust than those for the $\left[0.5 k_{ \pm i, R}, 2 k_{ \pm i, R}\right]$ range. However, it is still not clear what set of experimental or theoretical rate constants accurately describes the kinetics of the SARS-CoV RdRp complex.
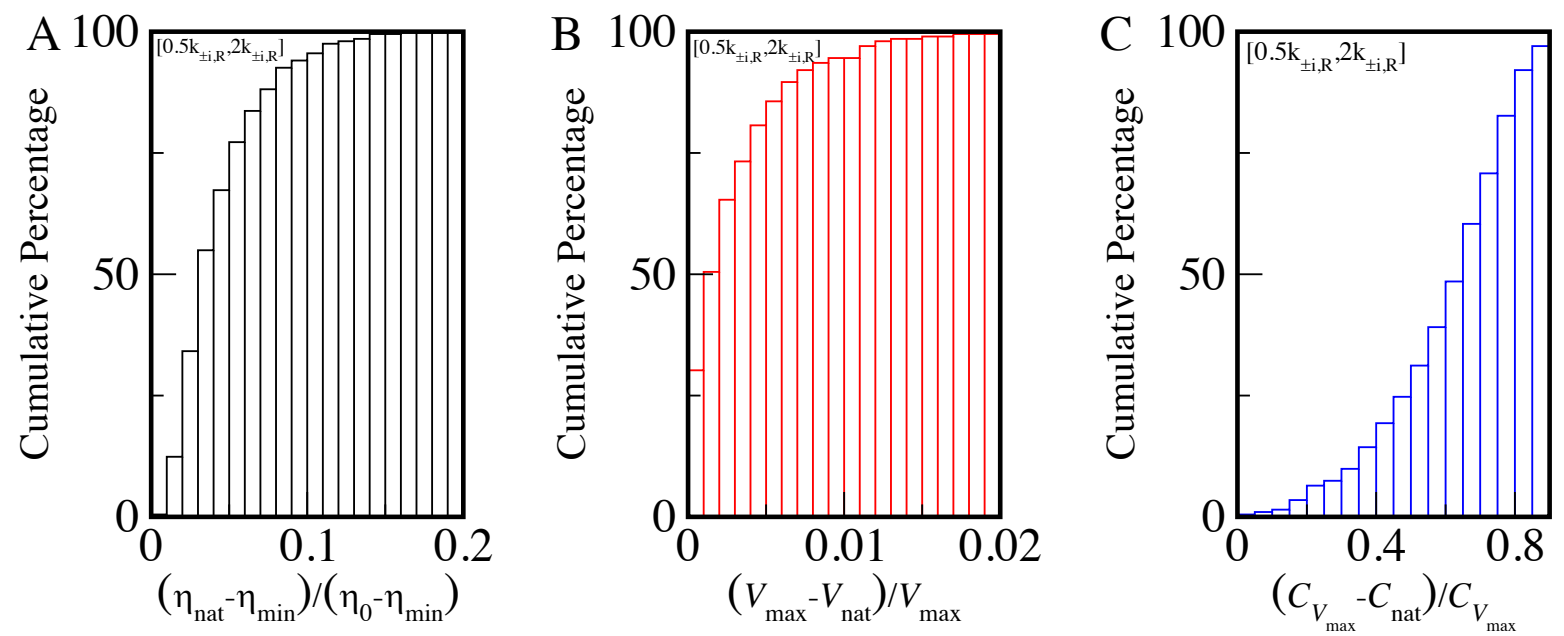

FIG. S4. Cumulative histograms from random sampling on the interval $\left[0.5 k_{ \pm i, R}, 2 k_{ \pm i, R}\right]$ showing the fraction of kinetic parameter sets that satisfy a particular inequality (see text). A. Difference between the native error rate $\eta_{\text {nat }}$ and the minimum error $\eta_{\min }$ versus the error rate $\eta_{0}$ with no proofreading (black bars). B. Difference between the maximum speed $V_{\text {max }}$ and the native speed $V_{\text {nat }}$ (red bars). C. Difference between the proofreading cost at the maximum speed $C_{V_{\max }}$ and the native cost $C_{\text {nat }}$ (blue bars). 

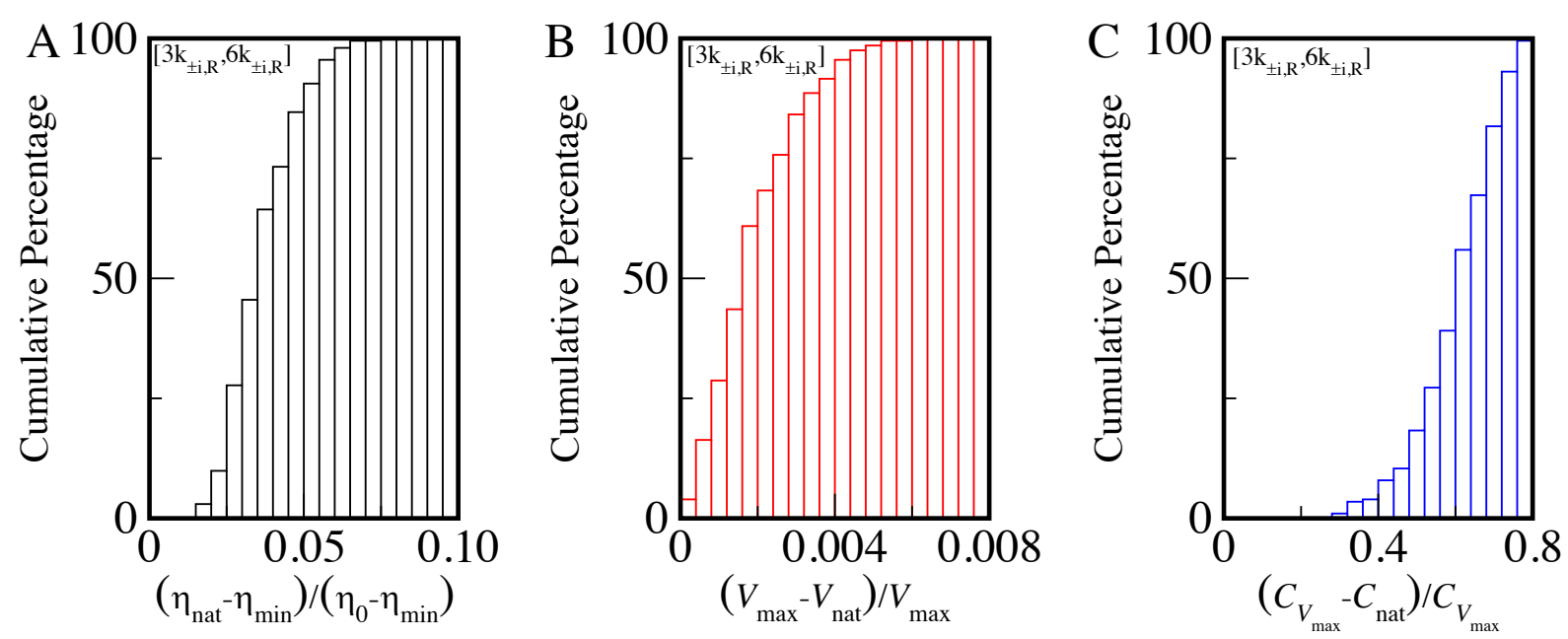

FIG. S5. Cumulative histograms from random sampling on the interval $\left[3 k_{ \pm i, R}, 6 k_{ \pm i, R}\right]$ showing the fraction of kinetic parameter sets that satisfy a particular inequality (see text). A. Difference between the native error rate $\eta_{\text {nat }}$ and the minimum error $\eta_{\min }$ versus the error rate $\eta_{0}$ with no proofreading (black bars). B. Difference between the maximum speed $V_{\max }$ and the native speed $V_{\text {nat }}$ (red bars). C. Difference between the proofreading cost at the maximum speed $C_{V_{\max }}$ and the native cost $C_{\text {nat }}$ (blue bars).

\section{INTERPLAY OF THE PROBABILITIES WITH THE POL-EXO SLIDING RATE}

To gain additional insight into why the speed $V$ of the SARS-CoV RdRp complex displays a non-monotonic dependence on the Pol-Exo sliding rate $k_{2, R}$ in Fig. $2 \mathrm{~B}$ in the main text, we examined the dependence of the probabilities of all five of the biochemical states on the Pol-Exo sliding rate. To this end, Fig. S6 shows how the probabilities $P_{i}$ $(i=E, E R, E R *, E W, E W *)$ of the biochemical states on the KPR mechanism depend on the Pol-Exo sliding rate. Specifically, the speed $V=k_{p, R} P_{E R}$, which is proportional to the probability $P_{E R}$, decreases as the Pol-Exo sliding rate $k_{2, R}$ gets higher due to increases in the probabilities $P_{E}$ and $P_{E R *}$. On the left-hand side of the maximum in $P_{E R}$, the speed decreases as the Pol-Exo sliding rate $k_{2, R}$ gets lower due to an increase in the probability $P_{E W}$. Therefore, changes in the probabilities of biochemical states other than $E R$ are responsible for the non-monotonic dependence of the probability $P_{E R}$, and hence the speed $V$, on the Pol-Exo sliding rate of the SARS-CoV RdRp complex. 


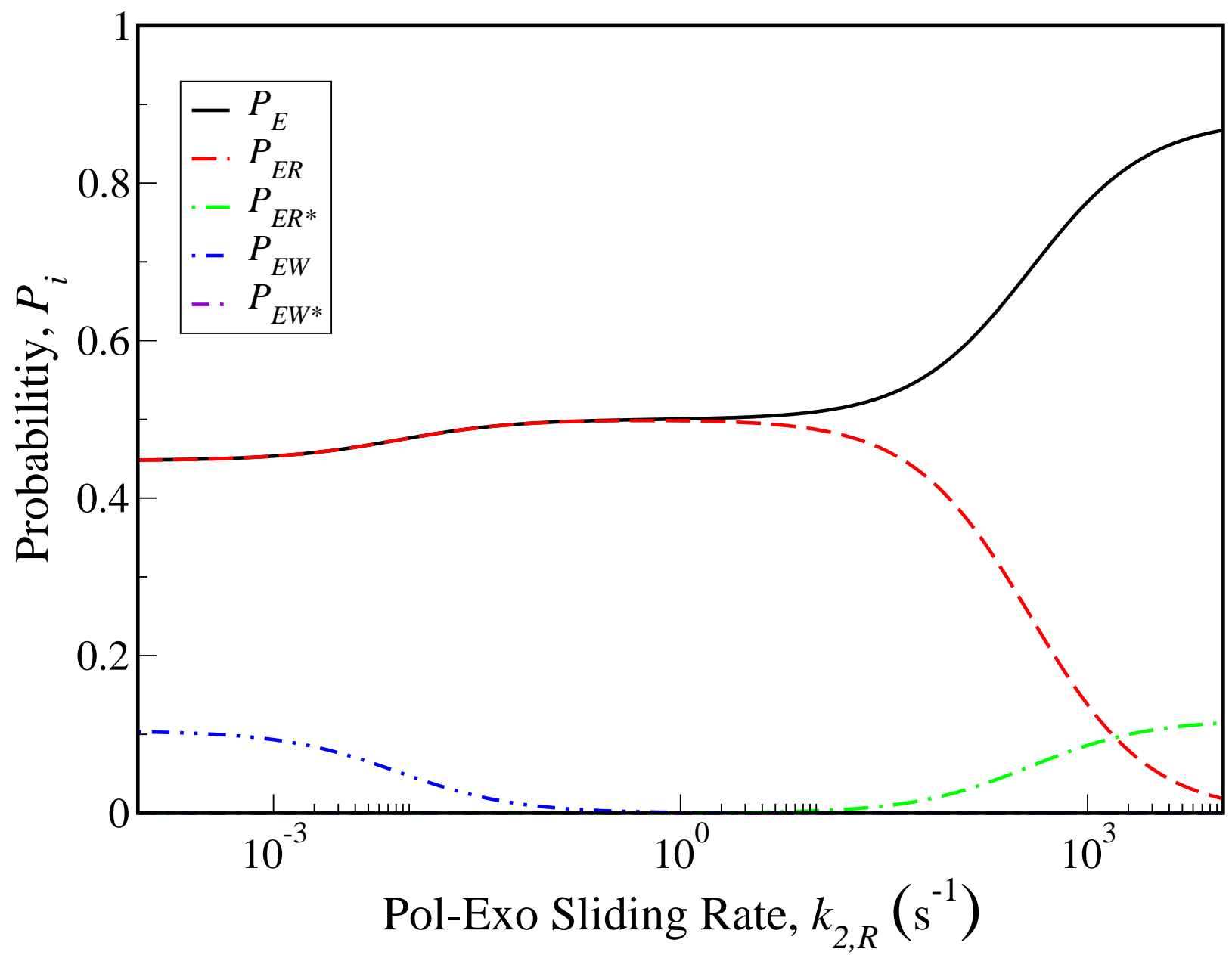

FIG. S6. Dependence of the probabilities $P_{i}(i=E, E R, E R *, E W, E W *)$ of all five of the biochemical states on the KPR mechanism with the Pol-Exo sliding rate $k_{2, R}$. Note that the probability $P_{E W *}$ is very small (on the order of $10^{-6}-10^{-5}$ ) and is not visible on this plot.

\section{BIOINFORMATICS CALCULATIONS: MUTATION PROBABILITY AND EFFECTIVE SPEED}

The BJ01 genome[3] for SARS-CoV was used to investigate how the changes in the error rate $\eta$ may affect the rate of production of functional virus copies, namely, the effective speed $V_{\text {eff }}$. The 22 open read frames (ORFs) in the BJ01 genome have been well defined in $\mathrm{Xu}$ et al.[2], allowing us to divide each ORF into non-overlapping codons and to examine the severity of each potential mutation in the corresponding amino acid. Such severity was measured by calculating the distance between the amino acid before and after the mutation 
using the BLOSUM62 matrix[11]. BLOSUM gives low values to a pair of amino acids when they are not homologous. We reasoned that a non-homologous amino acid can result in a protein with a different structure that further alters its functionality and ultimately leads to a non-functional virus copy. We used BLOSUM62 as it is a standard BLOSUM matrix which puts neither an extremely stringent nor an extremely loose constraint on the similarity of the amino acids. Thus, our analysis of the severity of the mutations in the amino acids can reflect most of the real changes in the functionality of the proteins.

\section{SARS-CoV Genome}

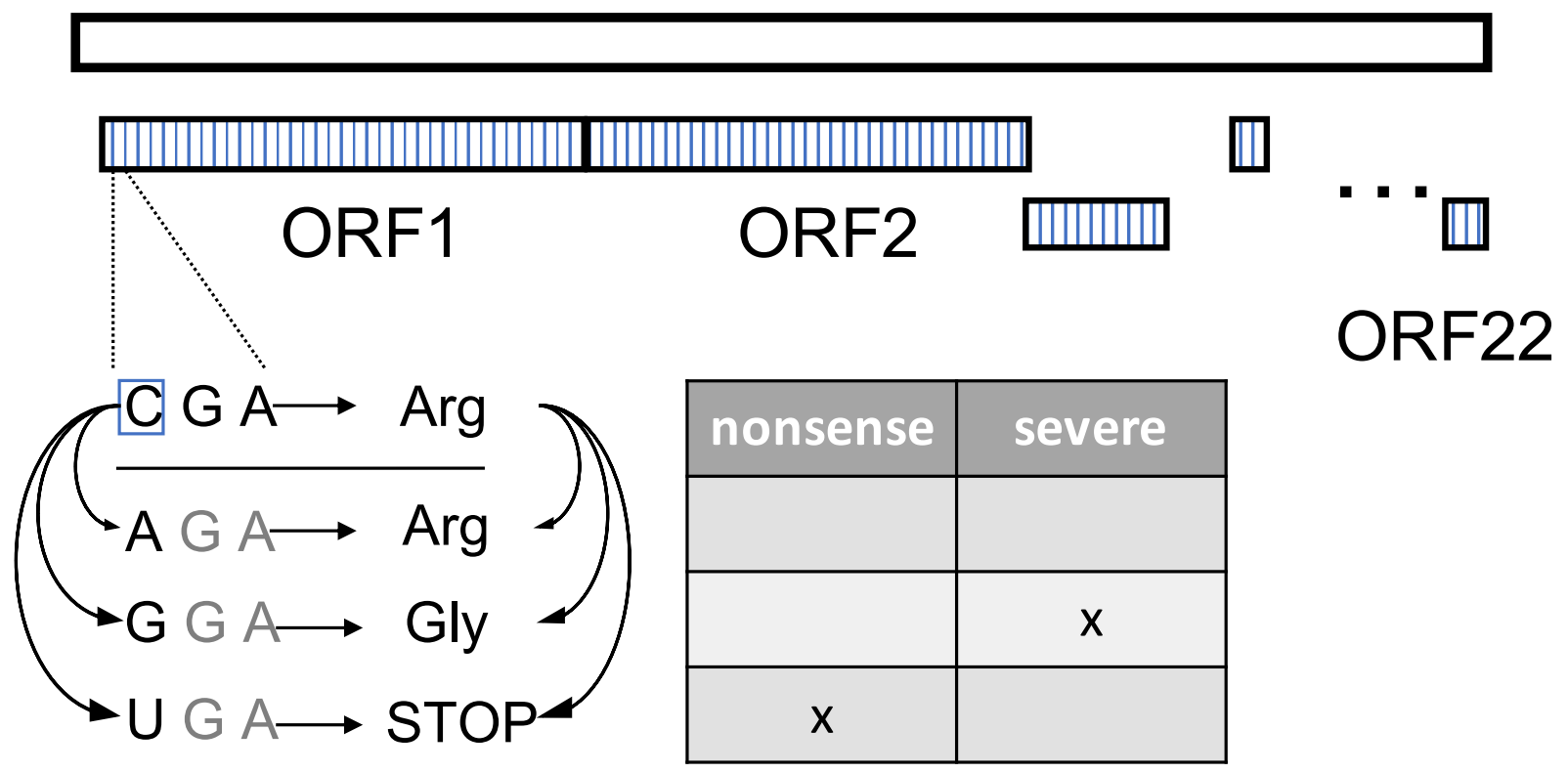

FIG. S7. Bioinformatics pipeline for identifying potential nonsense and severe missense mutations showing the segmentation of the SARS-CoV genome into 22 open reading frames (ORFs) and codons. Nonsense mutations change the original codon to a stop codon, while severe missense mutations change the identity of the amino acid, e.g., from arginine (Arg) to glycine (Gly).

The complete bioinformatics pipeline for identifying potential severe and nonsense mutations is shown in Fig. S7. We define any mutation resulting in an amino acid with a homologous distance $\leq-2$ from the original one as a "severe mutation". Note that we used -2 because we desired only severe mutations and because -2 is the smallest cutoff that renders us a nonempty set of severe mutations. This protocol enabled us to identify all severe mutations in the BJ01 genome and to calculate the corresponding effective speed of the 
SARS-CoV RdRp complex. Next, since a nonsense mutation is defined as a mutation leading to a premature stop codon, and hence, results in a severe change in the protein structure, we separately identified all nonsense mutations and evaluated their effect on the effective speed of the SARS-CoV RdRp complex. The purpose of this analysis on the nonsense mutations is to determine whether we can reach the same biological conclusion with a more stringent enrollment rule of the mutations that may lead to a non-functional virus copy. In our analysis, we also consider the probability of both nonsense and severe mutations together, namely, as "severe missense mutations".

Specifically, we calculated the fraction of non-functional virus copies, namely, the mutation probability $p$ using the following equation from the so-called complex formalism:

$$
p=1-\prod_{i}\left(1-n_{i} \eta\right) .
$$

Here, the product is taken over all nucleotide bases that could have a nonsense or a severe missense mutation, $n_{i}$ is the number of nonsense or severe missense mutations on the nucleotide base $i$ that may lead to a non-functional virus copy, and $\eta$ is the error rate. From this definition of the mutation probability $p$, the fraction of functional virus copies $1-p$ is determined using the equation:

$$
1-p=\prod_{i}\left(1-n_{i} \eta\right) .
$$

Using the fraction of functional virus copies $1-p$, we calculate the rate of production of functional virus copies, namely, the effective speed $V_{\text {eff }}$ of the SARS-CoV RdRp complex over the whole genome with $N=29725$ bases using the equation:

$$
V_{\mathrm{eff}}=\frac{V(1-p)}{N} .
$$

To understand how the effective speeds $V_{\text {eff }}$ calculated using the complex formalism compare to those determined using more a straightforward approach, we used a simple power law instead of the complex formalism to compute the effective speed $V_{\text {eff }}$. For the simple power law, $V_{\text {eff }}$ is calculated from the following equation:

$$
V_{\text {eff }}=\frac{V(1-\eta)^{N_{m}}}{N},
$$

where $N_{m}$ is the number of bases susceptible to nonsense or severe missense mutations, and $V, \eta$, and $N$ have the same definitions as they do in the complex formalism (see Eq. (S15)Eq. (S17)). The results shown in Fig. S8 are qualitatively similar for both the complex 
formalism and the simple power law. This gives us confidence that the $V_{\text {eff }}$ calculations using the complex formalism are robust.

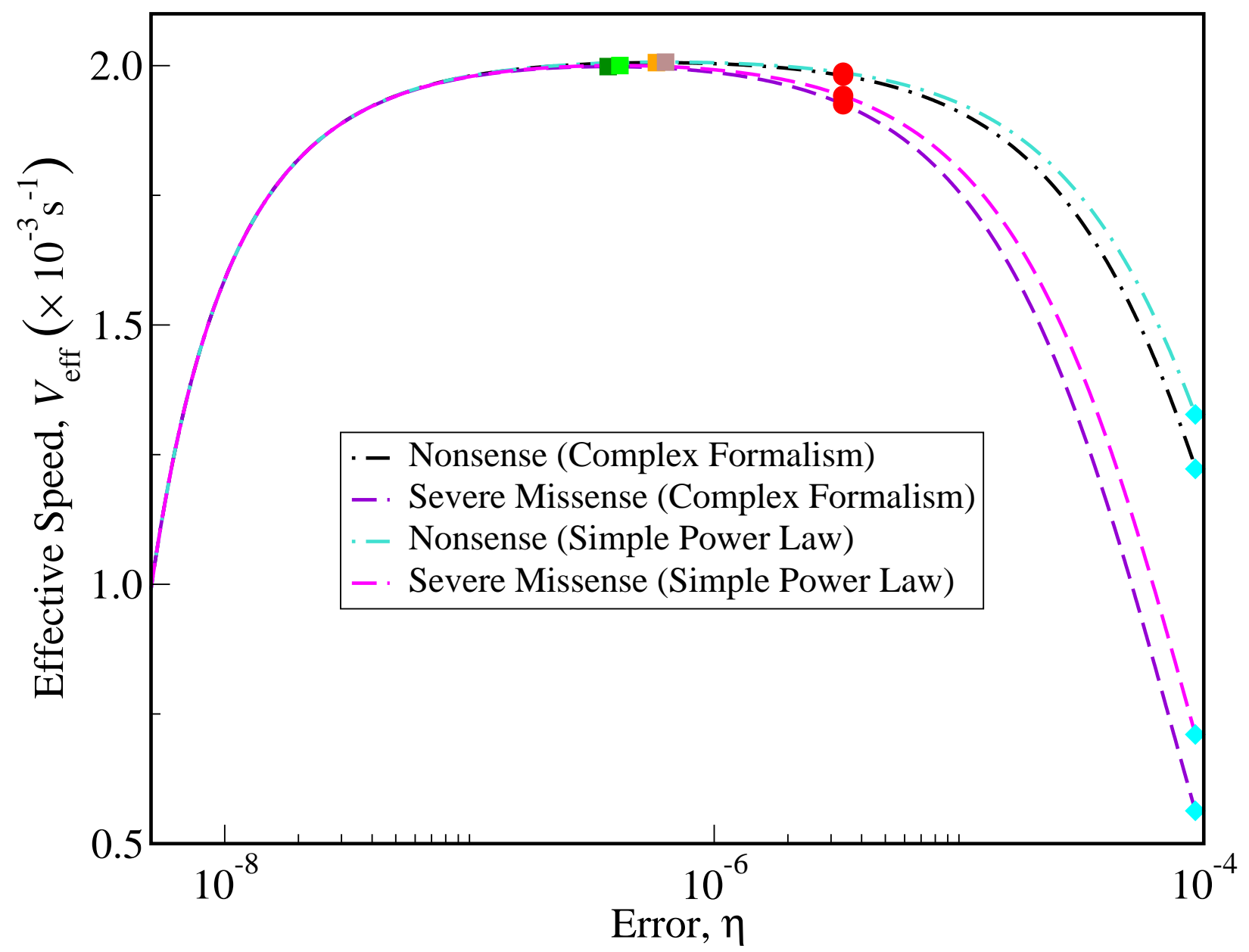

FIG. S8. Dependence of the effective speed $V_{\text {eff }}$ on the error rate $\eta$ for nonsense mutations (dasheddotted black and turquoise curves) and severe missense mutations (dashed violet and magenta curves) for the complex formalism and the simple power law (see plot legend). The red dot denotes the native system, the orange, brown, dark green, and green squares denote the maximum $V_{\text {eff }}$, and the cyan diamonds denote the system at low Pol-Exo sliding rate $k_{2, R} \sim 10^{-4} \mathrm{~s}^{-1}$.

Note that we could have also defined the speed as $V=J_{R}+J_{W}$ instead of $V=J_{R}$. However, because $J_{R}>>J_{W}$ the speed $V$ and the effective speed $V_{\text {eff }}$ computed using the two definitions above are indistinguishable from each other in Fig. S9A and B. Since $J_{W}$ is negligible in the definition of $V$, the effects of the nonsense and severe missense mutations are counted only once. 

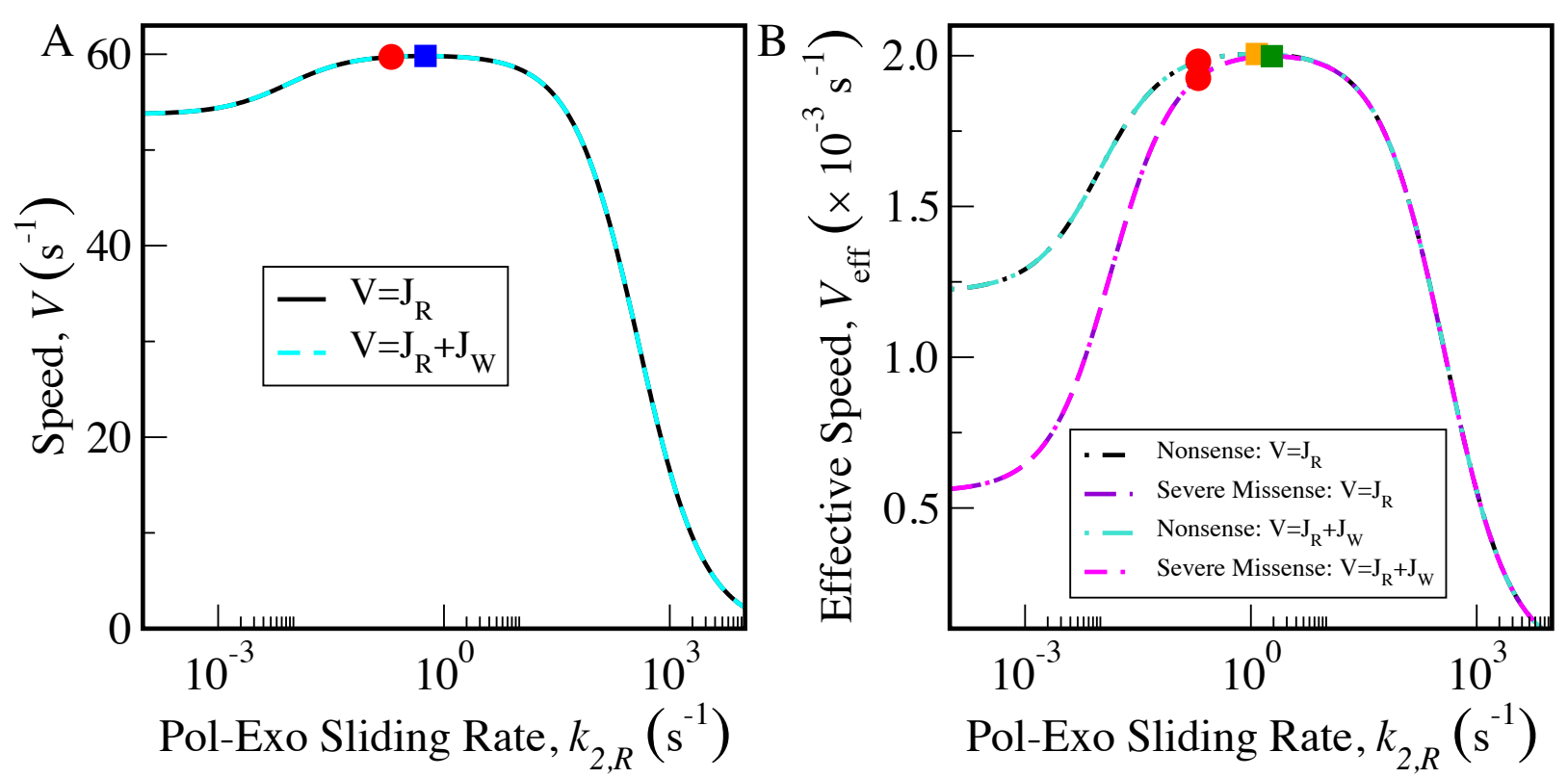

FIG. S9. The interplay of the speed with the Pol-Exo sliding rate for two different definitions of the speed $V=J_{R}$ and $V=J_{R}+J_{W}$. A. The speed $V$ as a function of the Pol-Exo sliding rate $k_{2, R}$. B. The effective speed $V_{\text {eff }}$ for nonsense and severe missense mutations as a function of the Pol-Exo sliding rate $k_{2, R}$. The curves of the speed and effective speed are indistinguishable for the two definitions of $V$ (see plot legend).

\section{INTERPLAY BETWEEN PROOFREADING COST AND EFFECTIVE SPEED}

To determine why the SARS-CoV RdRp complex does not reach the maximum effective speed, we analyzed the interplay between the effective speed $V_{\text {eff }}$ and the proofreading $\operatorname{cost} C$ due to variation of the Pol-Exo sliding rate $k_{2, R}$. The interplay between these two characteristic properties can be shown explicitly as a function of the Pol-Exo sliding rate as in Fig. 3A in the main text, or it can also be shown implicitly using a trade-off plot as in Fig. S10. One can see that the native system (red dot) lies on a non-trade-off branch of the effective speed-cost curve in Fig. S10. This means that any increase in the effective speed will inevitable lead to an increase in the proofreading cost. Specifically, the cost would increase by $\sim 10$-fold if the SARS-CoV RdRp complex increased its speed by $\sim 4 \%$ to reach the maximum effective speed (blue square). Thus, the SARS-CoV RdRp complex must pay a price in the proofreading cost in order to achieve the maximum effective speed. 


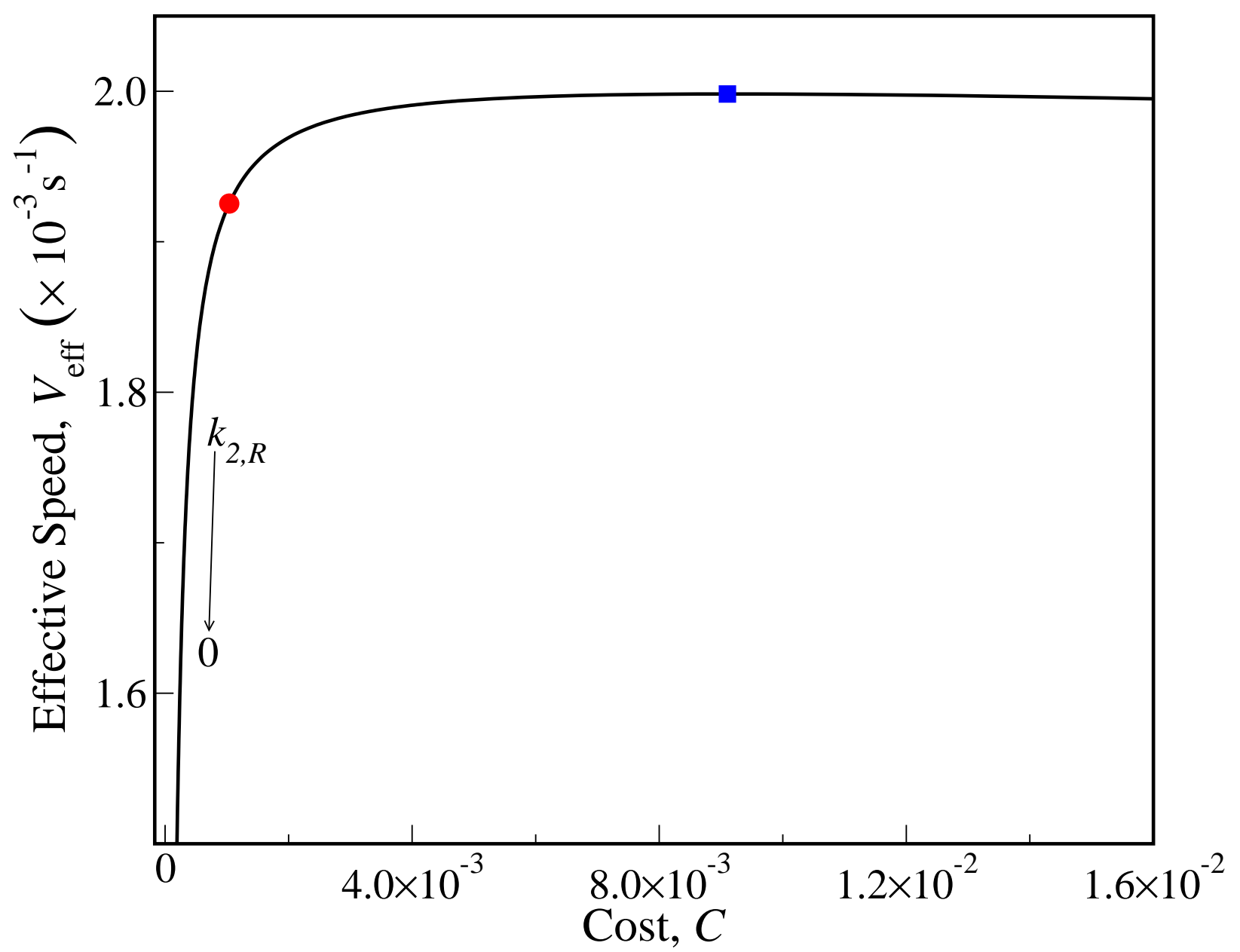

FIG. S10. The interplay of the effective speed $V_{\text {eff }}$ with the proofreading $\operatorname{cost} C$ shown using a trade-off plot. The red dot denotes the native system, and the blue square denotes the maximum $V_{\text {eff. }}$ 


\section{EFFECTIVE SPEED DISTRIBUTION}
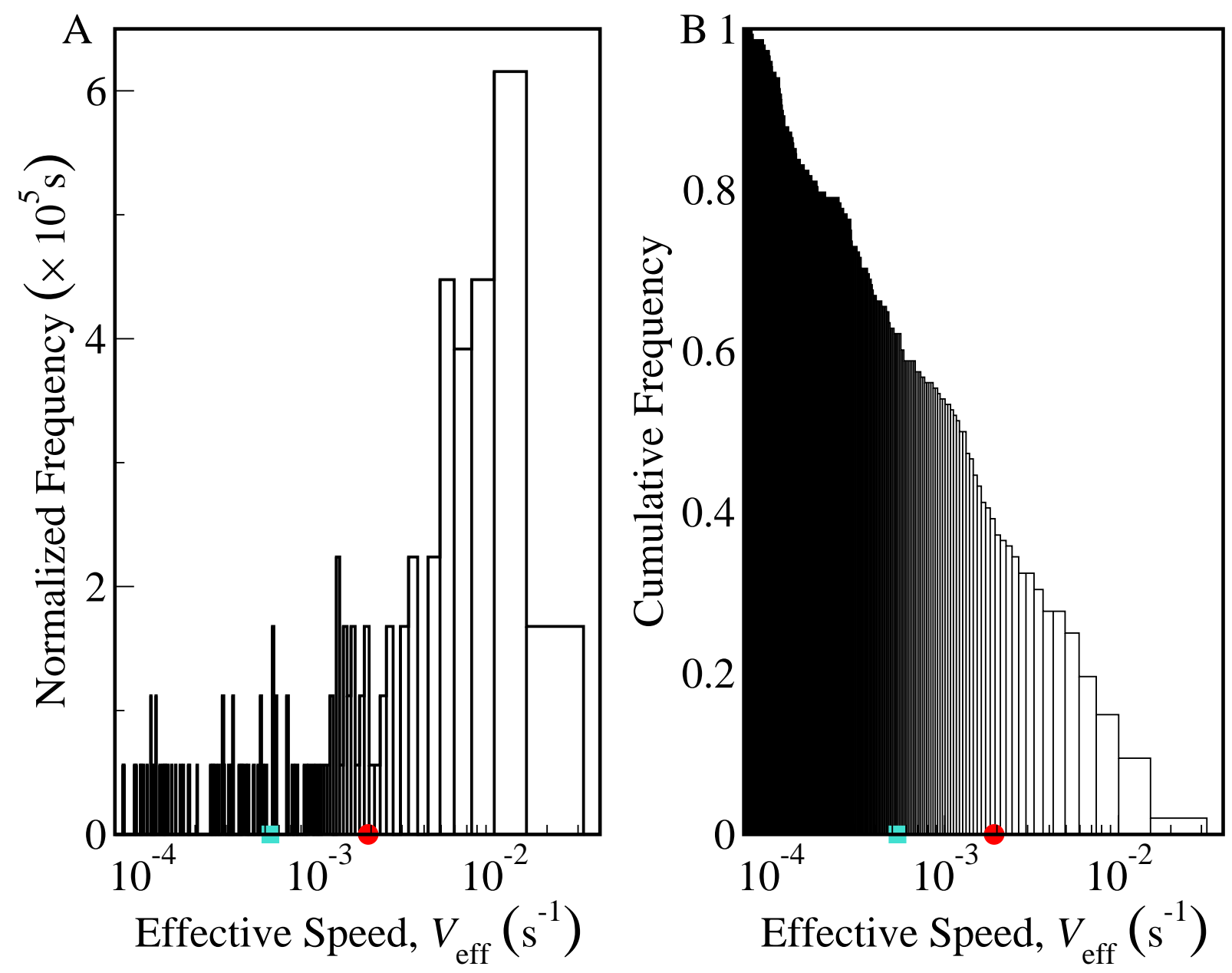

FIG. S11. Histograms of the effective speed $V_{\text {eff }}$ distribution for the SARS-CoV RdRp complex from variation of the polymerization rate $k_{1, R}=k_{p, R}$ and the Pol-Exo sliding rate $k_{2, R}$ at a fixed error rate $\eta_{\text {nat }}=3.4 \times 10^{-6}$. A. Normalized histogram of the $V_{\text {eff }}$ distribution for points corresponding to proofreading costs $C$ within $\sim 85 \%$ of the theoretical minimum cost. B. Cumulative histogram of the same $V_{\text {eff }}$ distribution. The red dot denotes the $\mathrm{V}_{\text {eff }}$ of the native system, and the turquoise square denotes the $\mathrm{V}_{\text {eff }}$ at the theoretical minimum cost. Note the log scale on the x-axis for $V_{\text {eff }}$ in both plots.

To better understand the interplay between the proofreading cost $C$ and the effective speed $V_{\text {eff }}$ shown in Fig. $3 \mathrm{C}$ in the main text, we looked at the effective speed distribution due to variation of both the polymerization rate $k_{1, R}=k_{p, R}$ and the Pol-Exo sliding rate $k_{2, R}$ for the error rate fixed at the native one $\eta_{\text {nat }}=3.4 \times 10^{-6}$. Fig. S11A shows the normalized 
$V_{\text {eff }}$ distribution for the SARS-CoV RdRp complex around the native system (red dot) and the theoretical minimum cost (turquoise square). The histrograms of the $V_{\text {eff }}$ distribution were generating using points within $\sim 85 \%$ of the minimum proofreading cost on the costerror bound (see Figs. 3B and 3C in the main text). Interestingly, there are many effective speeds in the $V_{\text {eff }}$ distribution that are either faster or slower than the native system and the speed at the theoretical minimum cost. Moreover, the cumulative $V_{\text {eff }}$ distribution in Fig. S11B shows that $\sim 40 \%$ of the effective speeds are slower than the native system and $\sim 70 \%$ of them are slower than the system at the theoretical minimum cost, respectively. Taking Fig. 3C in the main text into account, these results indicate that a decrease in the proofreading cost does not necessarily lead to an increase in the effective speed and vice versa. Therefore, our results show that the proofreading cost and the error rate are invariant to changes in the two kinetic parameters $k_{1, R}=k_{p, R}$ and $k_{2, R}$, but increasing the kinetic parameters (i.e., lowering the barriers between the biochemical states) leads to faster effective speeds of the SARS-CoV RdRp complex.

\section{RANKINGS THE CHARACTERISTIC PROPERTIES}

The results of our study on the interplay of the characteristic properties from single and global parameter sampling appear to indicate that while the speed is nearly maximized to optimized the rate of production of functional virus copies, the proofreading cost also appears to be very important. To gain further insight into the importance of the proofreading cost $C$ relative to the speed $V$ and the error rate $\eta$ for each kinetic parameter, we followed the protocol developed in Mallory et al.[10] and ranked the characteristic properties using the quantitative metric $d_{k, i}$, where $k$ denotes the rate constant being varied and $i$ is its corresponding numerical index. Specifically, the quantity $d_{k, i}$ measures how close the characteristic property of the native system is to its optimal value for each kinetic parameter $k_{ \pm i, R}$. The metric is given by the following equation:

$$
d_{k, i} \equiv-\log _{10}\left(\frac{\left|f\left(k_{0}\right)-f\left(k_{\max / \min }\right)\right|}{f\left(k_{\max }\right)-f\left(k_{\min }\right)}\right) ; f\left(k_{i}\right)=\eta, V, C .
$$

The scaled difference $d_{k, i}$ enables us to determine how close the value of the native system $f\left(k_{0}\right)$ is to its optimal value $f\left(k_{\max / \min }\right)$. We normalize this expression by the difference between the maximum or minimum $f\left(k_{\max } / \min \right)$ values to eliminate any bias from constant 
terms and then take the $-\log _{10}$. To determine the rankings, we varied all of the the different rate constants $k_{ \pm i, R}$ (except $k_{-3, R}$, which is determined from the chemical potential difference over the futile cycle) within a predetermined interval $\left[10^{-3} k_{0}, 10_{0}^{3}\right]$ centered around the native rate constant $k_{0}$. Thus, in our quantitative analysis of the rankings, the characteristic property with the highest $d_{k, i}$ value is the most important, while the property with the lowest $d_{k, i}$ value is the least important.
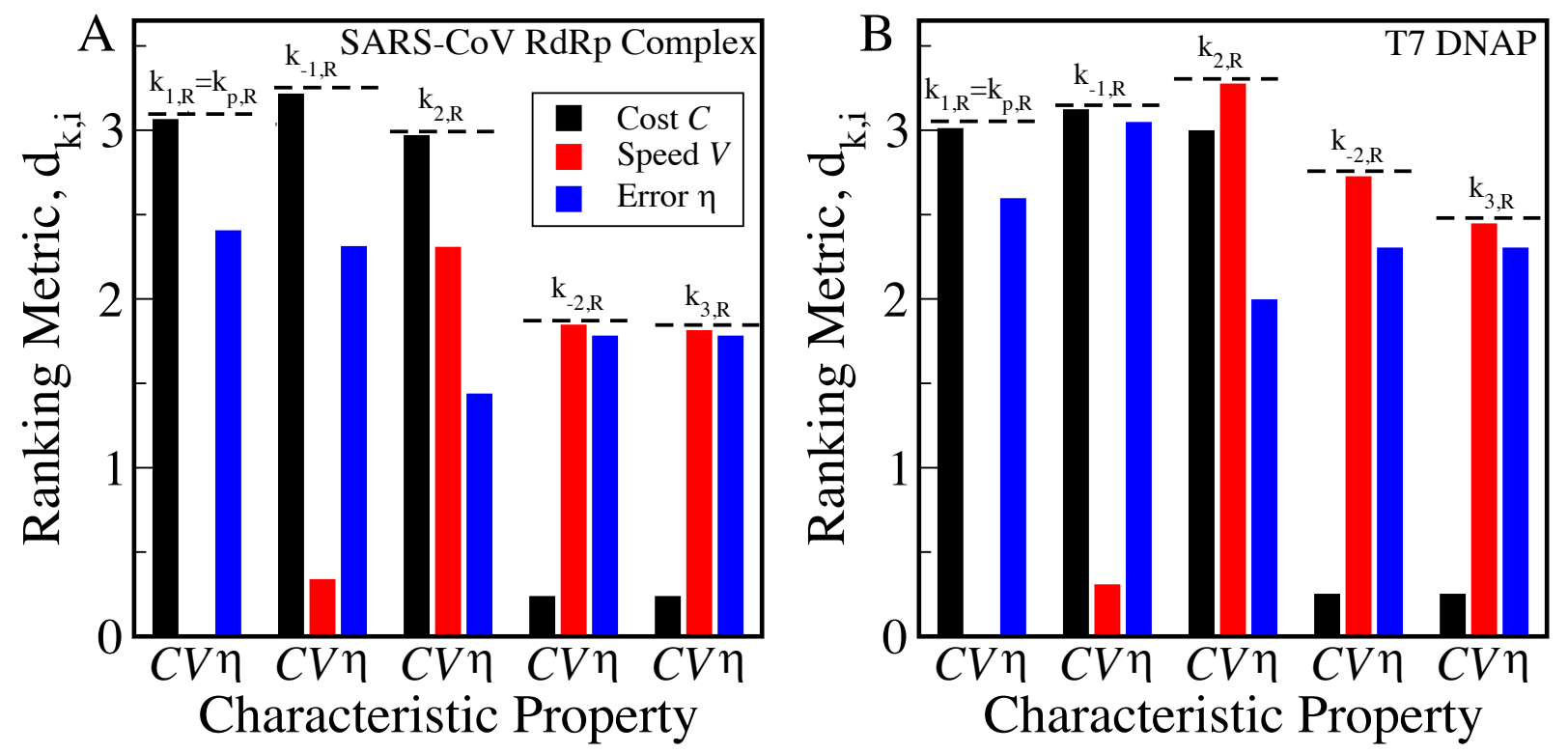

FIG. S12. Rankings $d_{k, i}$ (see text for definition) of the importance of the characteristic properties (proofreading cost $C$, speed $V$, and error rate $\eta$ ) for the SARS-CoV RdRp complex and T7 DNA polymerase for each kinetic parameter $k_{ \pm i, R}$. The kinetic parameters were varied individually on the interval $\left[10^{-3} k_{0}, 10^{3} k_{0}\right]$ centered around the native rate constant $k_{0}$. A. Rankings for the SARS-CoV RdRp complex. B. Rankings for the T7 DNA polymerase.

Fig. S12A shows the respective rankings from $d_{k, i}$ of each characteristic property for the SARS-CoV RdRp complex due to variation of each kinetic parameter individually on the aforementioned interval. Interestingly, the proofreading cost $C$ ranks first for $k_{1, R}=k_{p, R}$, $k_{-1, R}$, and $k_{2, R}$. However, the speed ranks first for $k_{-2, R}$ and $k_{3, R}$. Consequently, our quantitative study on the prioritization of the characteristic properties demonstrates that the proofreading cost is important for some steps, but the speed and the error rate are also quite important for other steps on the KPR mechanism. In comparison, Fig. S12B shows the same rankings for the T7 DNA polymerase. For this T7 DNA polymerase the rankings 
of the characteristic properties are all qualitatively the same except for $k_{2, R}$, where the speed ranks first and the cost comes in second.

\section{REFERENCES}

[1] J. Gunawardena, A linear framework for time-scale separation in nonlinear biochemical systems, PloS One 7, e36321 (2012).

[2] J. Xu, J. Hu, J. Wang, Y. Han, Y. Hu, J. Wen, Y. Li, J. Ji, J. Ye, Z. Zhang, et al., Genome Organization of the SARS-CoV, Genomics Proteomics Bioinformatics 1, 226 (2003).

[3] Q. Zhu, M. Yu, B. Fan, G. Chang, B. Si, W. Peng, T. Jiang, B. Liu, Y. Deng, H. Liu, et al., A Complete Sequence and Comparative Analysis of a SARS-associated virus (Isolate BJ01), Chin. Sci. Bull. 48, 941 (2003).

[4] S.-H. Yeh, H.-Y. Wang, C.-Y. Tsai, C.-L. Kao, J.-Y. Yang, H.-W. Liu, I.-J. Su, S.-F. Tsai, D.S. Chen, P.-J. Chen, et al., Characterization of severe acute respiratory syndrome coronavirus genomes in Taiwan: Molecular epidemiology and genome evolution, Proc. Natl. Acad. Sci. 101, $2542(2004)$.

[5] A. Shannon, B. Selisko, J. Huchting, F. Touret, G. Piorkowski, V. Fattorini, F. Ferron, E. Decroly, C. Meier, B. Coutard, et al., Rapid incorporation of Favipiravir by the fast and permissive viral RNA polymerase complex results in SARS-CoV-2 lethal mutagenesis, Nat. Commun. 11, $1(2020)$.

[6] J. J. Arnold and C. E. Cameron, Poliovirus RNA-dependent RNA polymerase (3Dpol): presteady-state kinetic analysis of ribonucleotide incorporation in the presence of $\mathrm{Mg}^{2+}$, Biochemistry 43, 5126 (2004).

[7] I. Wong, S. S. Patel, and K. A. Johnson, An induced-fit kinetic mechanism for dna replication fidelity: direct measurement by single-turnover kinetics, Biochemistry 30, 526 (1991).

[8] K. Banerjee, A. B. Kolomeisky, and O. A. Igoshin, Elucidating interplay of speed and accuracy in biological error correction, Proc. Natl. Acad. Sci. USA 114, 5183 (2017).

[9] K. Banerjee, A. B. Kolomeisky, and O. A. Igoshin, Accuracy of Substrate Selection by Enzymes is Controlled by Kinetic Discrimination, J. Phys. Chem. Lett. 8, 1552 (2017).

[10] J. D. Mallory, A. B. Kolomeisky, and O. A. Igoshin, Trade-Offs between Error, Speed, Noise, and Energy Dissipation in Biological Processes with Proofreading, J. Phys. Chem. B 123, 
4718 (2019).

[11] S. Henikoff and J. G. Henikoff, Amino acid substitution matrices from protein blocks, Proc. Natl Acad. Sci. USA 89, 10915 (1992). 